\title{
Spatial Pattern and Influencing Factors of Intangible Cultural Heritage of Music in Xiangxi, Central China
}

\section{xian yong zhang}

Jishou University

hui xiang ( $\nabla$ xhui_123@163.com )

Southwest University

Rui Liu

Chaohu University

\section{Research Article}

Keywords: intangible cultural heritage of music, spatial pattern, influencing factors, Xiangxi, utilization and protection

Posted Date: January 7th, 2022

DOI: https://doi.org/10.21203/rs.3.rs-1193841/v1

License: (c) (i) This work is licensed under a Creative Commons Attribution 4.0 International License. Read Full License

Version of Record: A version of this preprint was published at Heritage Science on March 21st, 2022. See the published version at https://doi.org/10.1186/s40494-022-00672-y. 


\title{
Spatial pattern and influencing factors of intangible cultural
} heritage of music in Xiangxi, central China

Xian-yong Zhang ${ }^{1}$, Hui Xiang ${ }^{2, *}$, Rui Liu ${ }^{3}$

1 School of Music and Dance, Jishou University, Jishou 416000, China. Email: langjuanyunshu@163.com.

2 College of Geographical Science, Southwest University, Chongqing 400715, China. Email: xhui_123@163.com.

3 School of Tourism Management, Chaohu University, Hefei 238000, China. Email: Liur18@1zu.edu.cn

\begin{abstract}
Intangible cultural heritage of music (ICHM) is an important part of intangible cultural heritage (ICH), and research on ICHM has extremely important theoretical value and practical significance. In this study, we aimed to analyze the spatial distribution characteristics of ICHMs and explore the main factors affecting their distribution in Xiangxi, Central China. Methods such as the nearest neighbor index, kernel density estimation, and buffer analysis were used in this study. The main conclusions are as follows: 1) the numbers of traditional music, traditional dance, traditional drama and quyi are 33,34, 30 and 14, respectively, indicating that quyi is relatively lacking in Xiangxi; 2) the distribution of ICHMs in Xiangxi have spatial heterogeneity, and the main trend is agglomeration. The national ICHMs, provincial ICHMs and municipal ICHMs are mainly concentrated in the south, the west, and the central and northern regions, respectively. The high-density areas of traditional music and traditional dance are located in the south, of traditional drama in the west, and of quyi in the north. 3) The distribution of ICHMs is affected by four factors: namely rivers, topography, GDP and transportation. The $0-3 \mathrm{~km}$ buffer zone of the rivers, with $100 \%$ of national ICHMs, $83.33 \%$ of provincial ICHMs and $84.52 \%$ of municipal ICHMs, is the centralized distribution area of ICHMs. $100 \%$ of the national ICHMs, $94.44 \%$ of the provincial ICHMs and $91.67 \%$ of the municipal ICHMs are distributed in hilly (200$500 \mathrm{~m}$ ) and low mountain areas (500-1000 m). Highways have the greatest impact on the distribution of ICHMs, followed by railways and ordinary roads. The economy has a significant positive impact on ICHM programs; the more developed the economy, the more ICHM projects. The results of this study can provide useful references for the theoretical research and practice management of $\mathrm{ICH}$.
\end{abstract}

Keywords: intangible cultural heritage of music; spatial pattern; influencing factors; 
Xiangxi; utilization and protection

\section{Introduction}

Intangible cultural heritage ( $\mathrm{ICH}$ ) has important social, history, aesthetics, artistic, science and cultural values [1], and it is the spiritual treasure and "living" representation of national culture $[2,3]$. ICH of music (ICHM) is an important part of ICH, and it was formed in a specific historical period. Furthermore, it has the characteristics of times, uniqueness, nationality, regional, and so on. Moreover, its inheritance and protection are difficult due to its scarcity and uniqueness. Therefore, research on ICHM has extremely important theoretical value and practical significance. Such efforts include improving the relevant theories of cultural research and promoting the prosperity and rejuvenation of national culture.

With the increasing attention around the world to $\mathrm{ICH}$, research on $\mathrm{ICH}$ has gradually become the core of academic circles [3-6]. Previous studies have examined $\mathrm{ICH}$ from the following three perspectives. First, the concept and categories of ICH have been clarified. ICH is the schematic diagram of lived practice, the creation of the heritage-maker [3]. Currently, ICH has been divided into 10 types [7] in China. These are folk literature, traditional music, traditional dance, traditional drama, quyi, folk custom, traditional art, traditional handicraft, traditional medicine, and sports recreation competition. Second, the research contents of ICH included innovative educational interventions [8], protection modes [3], evaluation of value [9], incentive mechanisms and srategy choices [5]. A few studies have discussed the spatial distribution and protection strategies of ICH worldwide [10]; however, there is no research on its influencing factors. Third, the research methods were diversed. For example, literature analysis [11], investigation and interview [3, 12], case demonstration [13], and scoring quantification methods [9], few studies have used spatial analysis and spatial mapping methods. Studies of ICH are conducive to the development and protection of ICH from the integrity. Nevertheless, they are unable to give local and subtle guidance to ICHM. Moreover, studies on ICHM have rarely been addressed, and the spatial pattern, as well as the influencing factors, have not been fully explored.

In view of this, this manuscript conducted an empirical research in Xiangxi, which is a mountainous area, a minority gathering area and a national cultural protection experimental area in China. The spatial pattern of ICHM and its influencing factors were explored by spatial analysis methods and geographic mapping models. The research aims of the manuscript are to provide a basis for other scholars, give useful suggestions for the development and protection of $\mathrm{ICH}$, and contribute to cultural 
revitalization.

\section{Materials and methods}

\subsection{Research area}

Xiangxi (the full name is Xiangxi Tujia and Miao Autonomous Prefecture) lies in Hunan Province, central China (Fig. 1), covering a total area of $15462 \mathrm{~km}^{2}$. It is located at longitudes $109^{\circ} 10^{\prime}-110^{\circ} 22^{\prime} \mathrm{E}$ and latitudes $27^{\circ} 44^{\prime}-29^{\circ} 38^{\prime} \mathrm{N}$. There are eight administrative units, and the center is Jishou. The highest and lowest points in Xiangxi are $1742 \mathrm{~m}$ and $67 \mathrm{~m}$, respectively. According to its cultural and natural settings, it is a special area with a distinct minority culture. Xiangxi is an ethnic minority area, with more than $90 \%$ of the population being ethnic minorities and a long history of minority culture. It is also a national cultural protection experimental area and plays an extremely important role in the protection of ICH. In addition, it is a mountainous area, and the spread of minority culture is blocked by the closed natural environment.

Therefore, the distribution pattern and influencing factors of ICHMs in Xiangxi are different from those in other regions. Moreover, it is representative and typical as a study area, which can provide scientific references for the cultural development of similar regions.

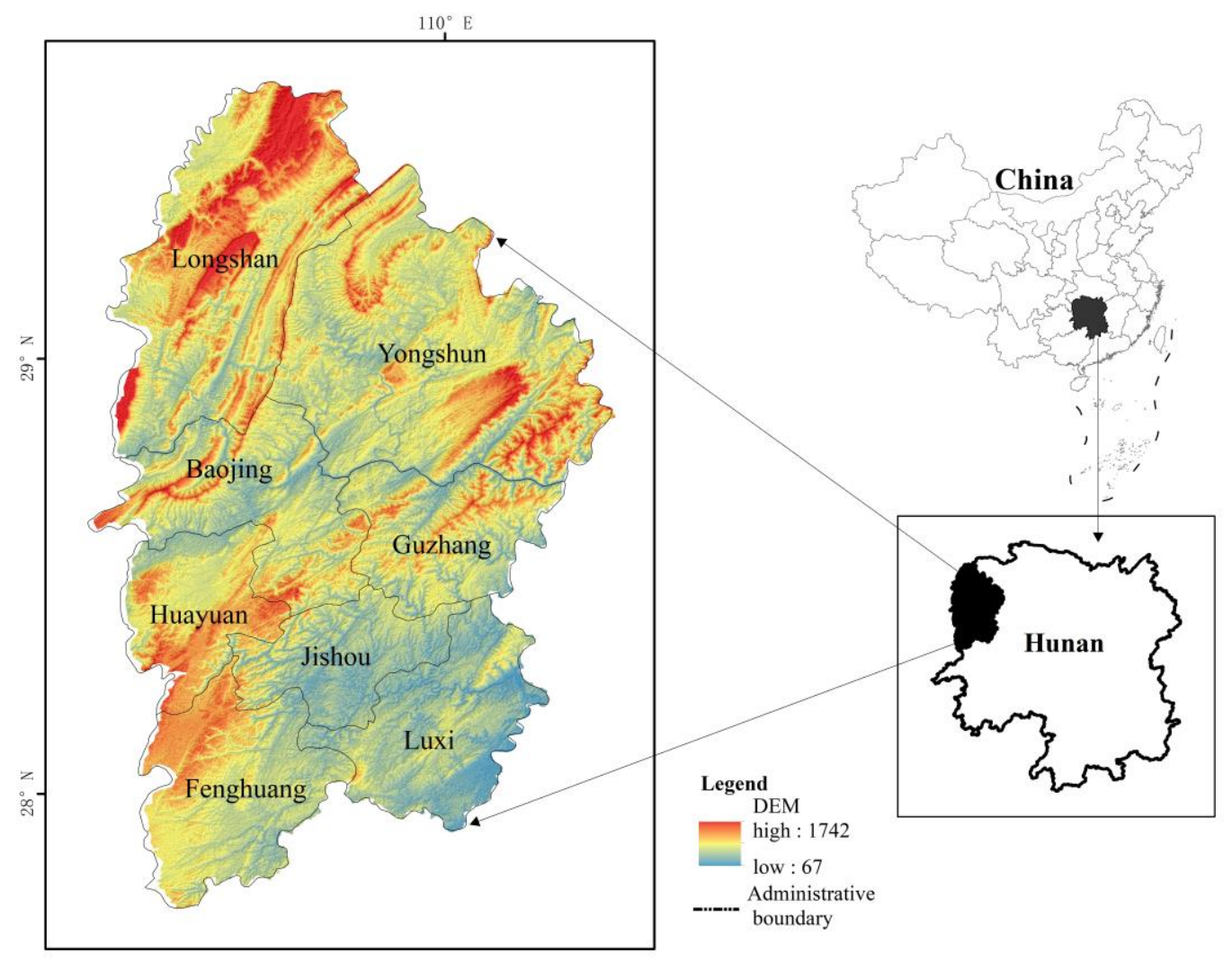

Fig. 1. The location of Xiangxi and its topographic features 


\subsection{Data sources}

The natural datasets (digital elevation model, i.e., DEM, water system) and socioeconomic datasets (administrative boundaries, ICHMs, transportation and GDP) were included in this paper. Among the natural datasets, DEM with a spatial resolution of $30 \mathrm{~m}$ was collected from the Resource and Environmental Science Data Center of the Chinese Academy of Sciences (http://www.resdc.cn/). The water system data were obtained from Google Map (http://www.gugeDitu.Net/). In the socioeconomic datasets, the administrative boundaries and traffic lines were derived from the national earth system science data sharing platform (http://www.geodata.cn/). ICHMs dataset were obtained from the China indigenous cultural heritage network (http://www.ihchina. cn/). The GDP database was from the statistical yearbook in Xiangxi (2019).

\subsection{Research methods}

\subsubsection{Graphic visualization of ICHMs}

Excel 2016 and ArcGIS 10.2 were used to realize graphic visualization of ICHMs. First, excel 2016 was used to count the batch, longitude, latitude and other attributes of ICHMs in Xiangxi. Second, the longitude and latitude dataset of ICHMs were transformed into maps based on ArcGIS 10.2. Third, color rendering was realized according to the attribute differences.

\subsubsection{The nearest neighbor index}

The nearest neighbor index tool of ArcGIS 10.2 was used to delimit the spatial distribution types of ICHMs (discrete, random, agglomeration), and it included two steps. First, the nearest distance was calculated according to the longitude and latitude of ICHMs [14]. Second, $Z$ and $P$ tests were carried out to verify the reliability of the model. The nearest neighbor index (NNI) was calculated as follows [15]:

$$
\begin{gathered}
N N I=\frac{\overline{D_{o}}}{\bar{D}_{e}} \\
\bar{D}_{e}=\frac{0.5}{\sqrt{n / A}}
\end{gathered}
$$

In the above formulas, the spatial points are discrete distribution if NNI is greater than 1 , randomly distributed equal to 1 , and agglomeration distribution less than $1 ; \overline{D_{o}}$ is the actual average nearest neighbor distance; $\bar{D}_{e}$ is the expected average distance; $n$ represents the number of observation points; $A$ is the total area of the study area.

The formula for $\mathrm{Z}$ test is as follows: 


$$
\begin{gathered}
Z=\frac{\overline{D_{o}}-\bar{D}_{e}}{S E_{d(\text { ran })}} \\
S E_{d(\text { ran })}=\frac{0.26162}{\sqrt{n^{2} / A}}
\end{gathered}
$$

In the above formulas, when $-1.96<Z<1.96$, it indicates that it has not passed the significance test [14]; when $Z>1.96$ or $Z<-1.96$, it is considered that the result is significant.

\subsubsection{Kernel density estimation}

Kernel density estimation has the advantages of three-dimensional, intuitive, simple and high precision [16-18], and it has been widely used in many fields [19-22]. Consequently, kernel density estimation was adopted to evaluate the density of ICHMs, and its calculation formula is as follows:

$$
P_{i}=\frac{1}{n \pi R^{2}} \times \sum_{n}^{j=1} K_{j}\left(1-\frac{D_{i j}^{2}}{R^{2}}\right)^{2}
$$

where $P_{i}$ is the density of point $i ; K_{j}$ is the weight of observation point $j ; D_{i j}$ is the distance between point $i$ and observation point $j ; R$ is the smoothing constant or bandwidth; $R>D_{i j}$; and $n$ is the number of observation points within $R[23,24]$.

\subsubsection{Buffer analysis}

To reveal the spatial distribution relationship between the ICHMs and the rivers, $1 \mathrm{~km}, 3 \mathrm{~km}$ and $5 \mathrm{~km}$ buffer zones of the rivers are established by using the buffer analysis function of ArcGIS 10.2. The formula is as follows:

$$
B_{i}=\left\{x: d\left(x, U_{i}\right), R\right\}
$$

Where: $B_{i}$ is the buffer zone; $x$ is the observation point; $U_{i}$ is the river $i ; d$ is the distance from $x$ to the rivers; and $R$ is the radius.

\section{Results and analysis}

\subsection{The structure of ICHM in Xiangxi}

Music can refer to general music (i.e., vocal music, dance, drama, and quyi) and special music (i.e., vocal music). This study uses the first definition, and therefore, we divided ICHMs into four categories (i.e., traditional music, traditional dance, traditional drama, quyi). By the end of August 2020, the national, provincial and municipal ICHMs in Xiangxi were 9, 18 and 84, respectively (Table 1). 
Table 1. The statistics of ICHMs in Xiangxi (items)

\begin{tabular}{ccccc}
\hline Categories & $\begin{array}{c}\text { National } \\
\text { ICHMs }\end{array}$ & $\begin{array}{c}\text { Provincial } \\
\text { ICHMs }\end{array}$ & $\begin{array}{c}\text { Municipal } \\
\text { ICHMs }\end{array}$ & The total \\
\hline Traditional music & 5 & 5 & 23 & 33 \\
Traditional dance & 3 & 6 & 25 & 34 \\
Traditional drama & 1 & 5 & 24 & 30 \\
Quyi & 0 & 2 & 12 & 14 \\
The total & 9 & 18 & 84 & 111 \\
\hline
\end{tabular}

The national ICHMs are mainly composed of traditional music and traditional dance, while traditional drama and quyi are relatively rare. The numbers of traditional music and traditional dance are 5 and 3 , accounting for $55.56 \%$ and $33.33 \%$ of the total, respectively. The quantities of traditional drama and quyi are 1 and 0 , accounting for $11.11 \%$ and $0 \%$, respectively.

The structures of provincial and municipal ICHMs are similar; that is, they are dominated by traditional music, traditional dance and traditional drama. For the provincial ICHMs, the numbers of the three are 5, 6, and 5, accounting for $27.78 \%, 33.33 \%$ and $27.78 \%$ of the total, respectively. The rest $11.11 \%$ ( 2 items) are quyi. For the municipal items, the quantities of the dominant three are 23, 25, and 24, and the proportions are $27.38 \%, 29.76$ and $28.57 \%$, respectively. The number of quyi is 12 , accounting for only $14.29 \%$ of the total.

On the whole, traditional music, traditional dance and traditional drama are abundant (the quantities are 33, 34 and 30, and the proportions are $29.73 \%, 30.63 \%$ and $27.02 \%$ of the total), while quyi is relatively lacking (the quantity is 14 , while the proportion is $12.62 \%$ ). The reason is that the inheritance of quyi mainly depends on teaching from mouth to mouth among individuals, which is prone to loss, resulting in its scarcity. As a result, further excavation and in-depth protection are needed.

\subsection{The spatial distribution characteristics of ICHM in Xiangxi}

\subsubsection{The Spatial distribution types}

According to Table 2, the main spatial distribution trends of ICHMs in Xiangxi are agglomeration. Among them, the values of $N N I, Z$ and $P$ for national ICHMs are $1.6802,3.9039$ and 0 , respectively, indicating that they are typical discrete types. The $N N I$ of provincial ICHMs was less than $1(0.8020)$, but it did not pass the significance test ( $P$ was -1.670 , and $Z$ was 0.1080 ). The above data show that the provincial ICHMs tend to be randomly distributed. The NNI of provincial ICHMs is less than $1(0.5543)$, and it passes the significance test ( $P$ and $Z$ were -7.8153 and 0 , respectively), indicating that the spatial distribution of the municipal ICHMs has a typical agglomeration characteristic. 
The NNIs of traditional music, traditional dance and traditional drama are less than 1 (between 0.4 and 0.7 ), and all have passed the significance test, indicating that the above three are concentrated distributions. The NNI of quyi is greater than 1 (1.1480), but it does not pass the significance test, indicating that it is a random distribution.

Table 2. The nearest neighbor indexes and distribution types of ICHM in Xiangxi

\begin{tabular}{ccccccc}
\hline Categories & $\begin{array}{c}\text { The observed } \\
\text { value }(\mathrm{m})\end{array}$ & $\begin{array}{c}\text { The expected } \\
\text { value }(\mathrm{m})\end{array}$ & NNI & $Z$ & $P$ & $\begin{array}{c}\text { Distribution } \\
\text { types }\end{array}$ \\
\hline $\begin{array}{c}\text { National } \\
\text { ICHMs }\end{array}$ & 0.1641 & 0.0977 & 1.6802 & 3.9039 & 0.0000 & $\mathrm{D}$ \\
$\begin{array}{c}\text { Provincial } \\
\text { ICHMs }\end{array}$ & 0.0855 & 0.1066 & 0.8020 & -1.6070 & 0.1080 & $\mathrm{R}$ \\
$\begin{array}{c}\text { Municipal } \\
\text { ICHMs }\end{array}$ & 0.0328 & 0.0591 & 0.5543 & -7.8153 & 0.0000 & $\mathrm{~A}$ \\
$\begin{array}{c}\text { Traditional } \\
\text { music }\end{array}$ & 0.0385 & 0.0865 & 0.4448 & -6.1018 & 0.0000 & $\mathrm{~A}$ \\
$\begin{array}{c}\text { Traditional } \\
\text { dance }\end{array}$ & 0.0589 & 0.0878 & 0.6708 & -3.6725 & 0.0002 & $\mathrm{~A}$ \\
$\begin{array}{c}\text { Traditional } \\
\text { drama }\end{array}$ & 0.0643 & 0.0904 & 0.7113 & -3.0244 & 0.0025 & $\mathrm{~A}$ \\
Quyi & 0.1333 & 0.1161 & 1.1480 & 1.0594 & 0.2893 & $\mathrm{R}$ \\
\hline
\end{tabular}

Note: $\mathrm{D}$ is the discrete abbreviation, $\mathrm{R}$ is random and $\mathrm{A}$ is agglomeration.

\subsubsection{The spatial distribution differences}

According to Fig. 2, we find that the distribution of ICHMs in Xiangxi is highly heterogeneous.
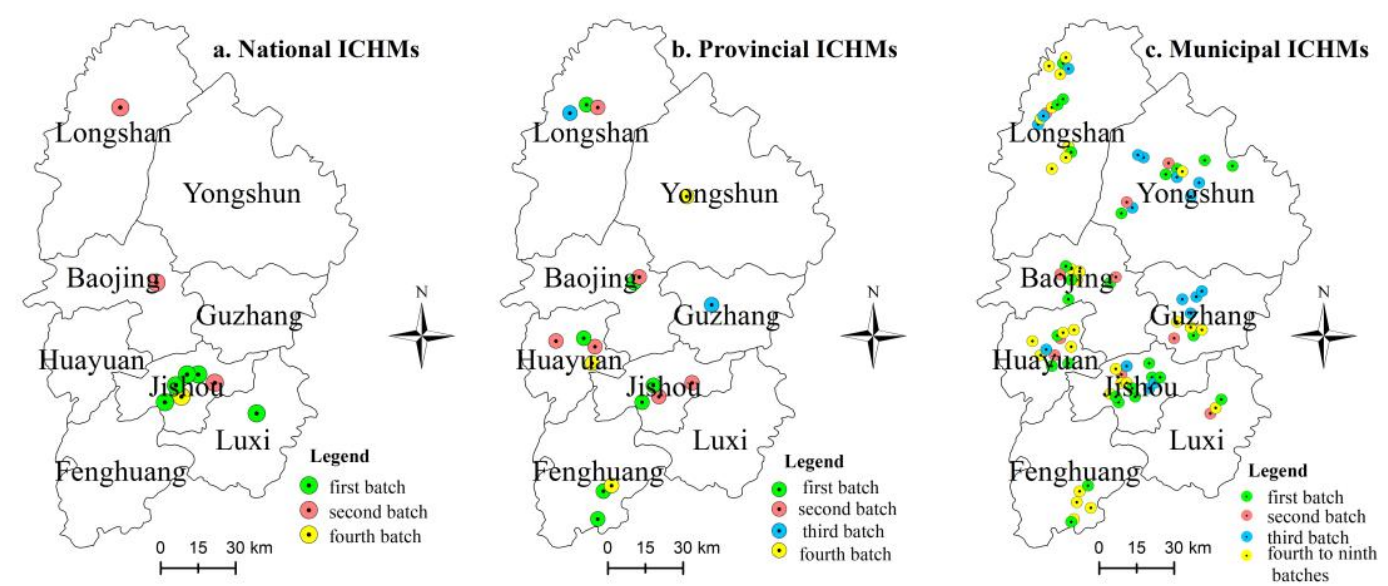

Fig. 2. The distribution of ICHMs in Xiangxi

Note: There are four batches of provincial and national ICHMs, and nine batches of municipal ICHMs. The fourth to ninth batches of municipal ICHMs were approved after 2012, which was the 
same time as the fourth batch of provincial and national ICHMs. Therefore, the fourth to ninth batches of municipal ICHMs were combined for the sake of comparison.

1) The national ICHMs are mainly concentrated in the south

The main parts of the first national ICHMs are traditional dance, and there are five projects, four in Jishou and one in Luxi, all located in the south (Fig. 2a). All items of the second national ICHMs are traditional music, and there are three items in all, which are scattered throughout the region. The remaining item of the fourth national ICHM is distributed in Jishou. Overall, Jishou, the administrative center of Xiangxi, is the enrichment area of national projects. AS for the reasons, Jishou has a relatively developed economy, and it has more sufficient funds for the inheritance and protection of ICHMs.

2) The provincial ICHMs is dense in the west and sparse in the east.

The first batch of provincial ICHMs, with a total of 7 items, are mainly composed by traditional music and traditional drama, and scattered in five administrative units of the west (Fig. 2b). The main components of the second provincial ICHMs are traditional dance, with a total of 6 projects distributed in the northwest and central areas of Xiangxi. The third batch are consisted of traditional drama and traditional dance, one for each, located in Guzhang and Longshan, respectively. There are three types of the fourth batch (traditional drama, traditional music and quyi), one for each, distributed in Yongshun, Huayuan and Fenghuang respectively (Fig. 2b). On the whole, Jishou and Huayuan have the most provincial ICHMs (4 projects each), followed by Baojing, Longshan and Fenghuang (2-3 projects), while Yongshun, Guzhang and Luxi have the least (0-1 project).

3) Municipal ICHMs are concentrated in the central and northern regions

The first batch of municipal ICHMs mainly focus on traditional music, traditional dance and traditional drama, with a total of 27 projects. Except for 2 items distributed in the southeast (located in Guzhang and Luxi), the other 25 items are all distributed in the north and west (Fig. 2c). The main parts of the second municipal ICHMs are traditional music and traditional dance, with a total of 10 items scattered throughout the region. The third municipal ICHMs, with traditional dances accounting for the largest proportion and a total of 17 items, are concentrated in the central and northern areas of Xiangxi. There are 30 items from the fourth to the ninth batch, which are concentrated in the western region of Xiangxi. In addition, there is little difference in the number of traditional music, traditional dance, traditional drama and quyi (6-8 items). Overall, Fenghuang and Luxi (in the southern area of Xiangxi) are sparse areas of municipal ICHMs, while other administrative units (mainly located in the northern and central parts of Xiangxi) are dense areas. 


\subsubsection{The spatial aggregation characteristics of ICHMs}

1) The spatial aggregation characteristics of different levels

For the national ICHMs, there are a high-density circle in the south (located in Jishou) and two subhigh-density circles in the center and north (located in Baojing and Longshan) (Fig. 3a). For the provincial ICHMs, there is a high-density area in the center (between Jishou and Huayuan) and two subhigh-density areas in the north (located in Longshan) and the south (located in Fenghuang) (Fig. 3b). The only high-density area of municipal ICHMs, which is a long strip, is in the center (between Jishou and Huayuan), and two subhigh-density areas are in the north (located in Yongshun and Longshan) (Fig. 3c).
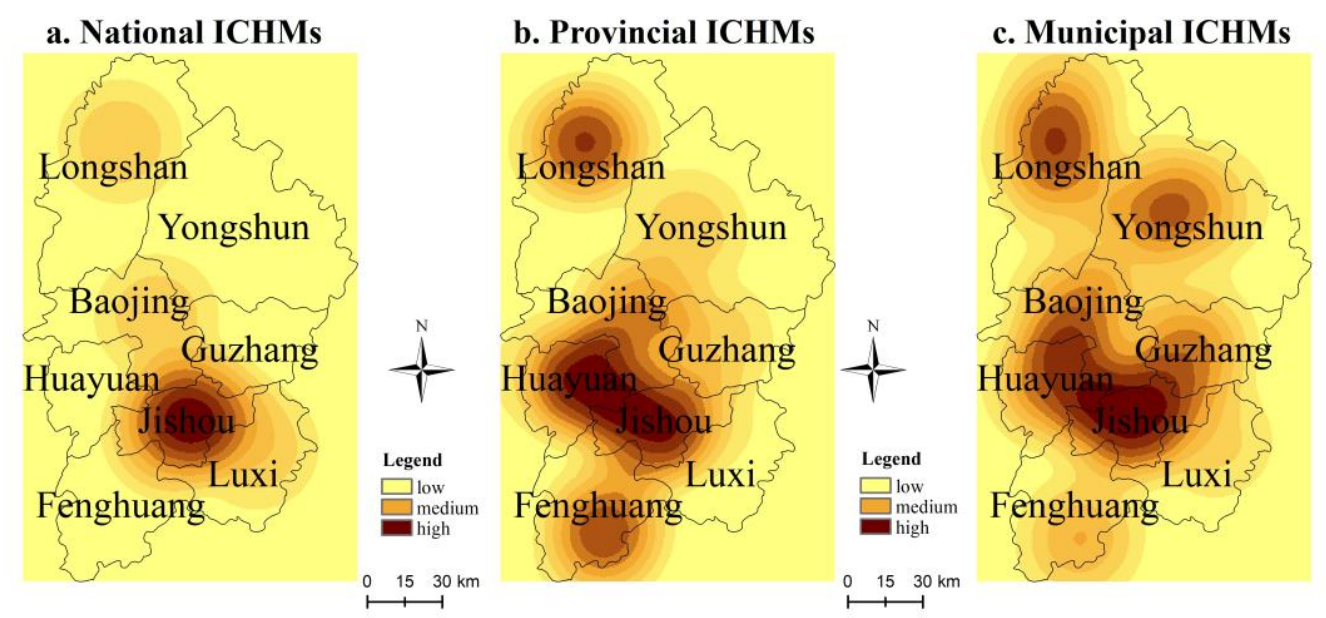

Fig. 3. The nuclear density distribution of different levels of ICHMs in Xiangxi

2) The spatial aggregation characteristics of different types

The density of traditional music is characterized by three subhigh-density areas (located in Longshan, Yongshun and Fenghuang) surrounding one high-density area (located in Jishou) (Fig. 4a). Traditional dance is concentrated in the south, and the density of which is consistent with the former, and the only one high-density area is located in Jishou and its surrounding areas (Fig. 4b). Traditional drama is concentrated in western Xiangxi, with two high-density areas and two subhigh-density areas. Among them, the two high-density areas are located in Longshan, the junction of Huayuan and Baojing, and the remaining two subhigh-density areas are located in Yongshun and Fenghuang (Fig. 4c). Quyi is concentrated in the north, and the two high-density areas are located in Yongshun and Longshan. The three subhigh-density areas are distributed in the centre and south, located in Guzhang, the junction of Baojing and Huayuan, and Fenghuang(Fig. 4d). 
a. traditional music

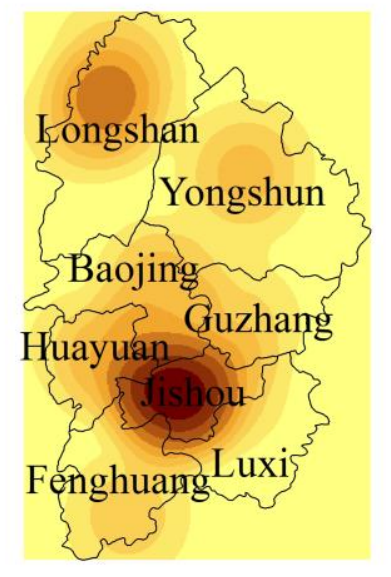

c. traditional drama

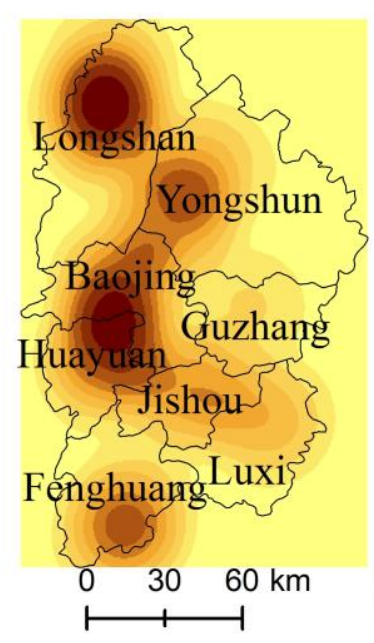

b. traditional dance

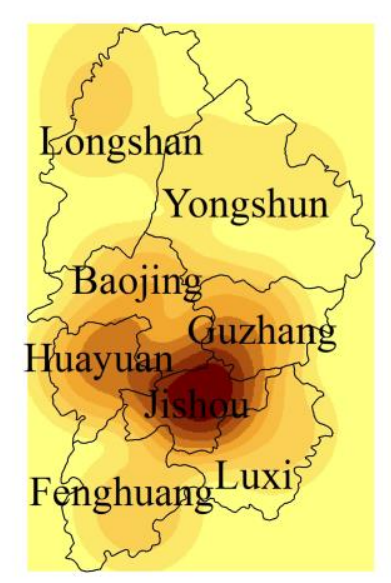

d. quyi

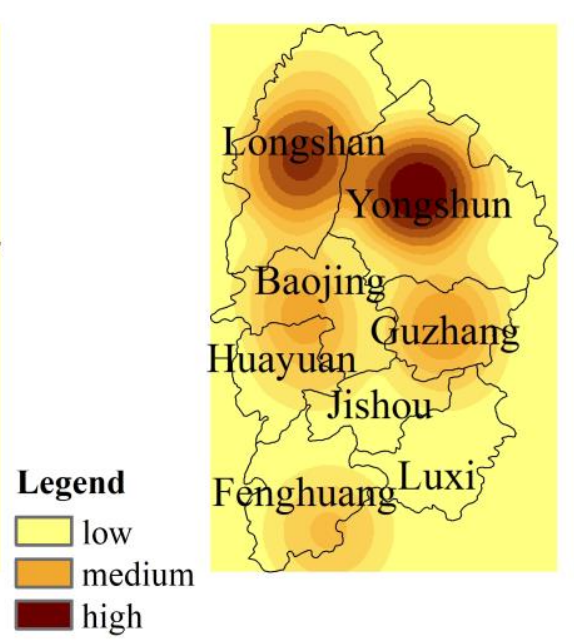

Fig. 4. The nuclear density distribution of different types of ICHMs in Xiangxi

\subsection{The influencing factors of ICHMs' spatial distribution}

\subsubsection{The influence of rivers on the distribution of ICHMs}

According to Fig. 5, ICHMs are concentrated near the rivers, indicating that rivers are important factors affecting the spatial distribution of ICHMs in Xiangxi. For national ICHMs, 3 items (accounting for 33.33\%) are distributed within the $0-1 \mathrm{~km}$ of the river, and 6 items (accounting for 66.67\%) are distributed within the 1-3 km (Table 3). The numbers of provincial ICHMs within $0-1 \mathrm{~km}, 1-3 \mathrm{~km}$ and $3-5 \mathrm{~km}$ of rivers are 7,8 and 3 , accounting for $38.89 \%, 44.44 \%$ and $16.67 \%$, respectively (Table 3 ). The numbers of municipal ICHMs within the above three buffer zones are 30, 41 and 13, accounting for $35.71 \%, 48.81 \%$ and $15.48 \%$, respectively (Table 3 ). In conclusion, the $0-3 \mathrm{~km}$ of the river is the centralized distribution area of ICHMs, with $100 \%$ national ICHMs, $83.33 \%$ provincial ICHMs and $84.52 \%$ municipal ICHMs. 

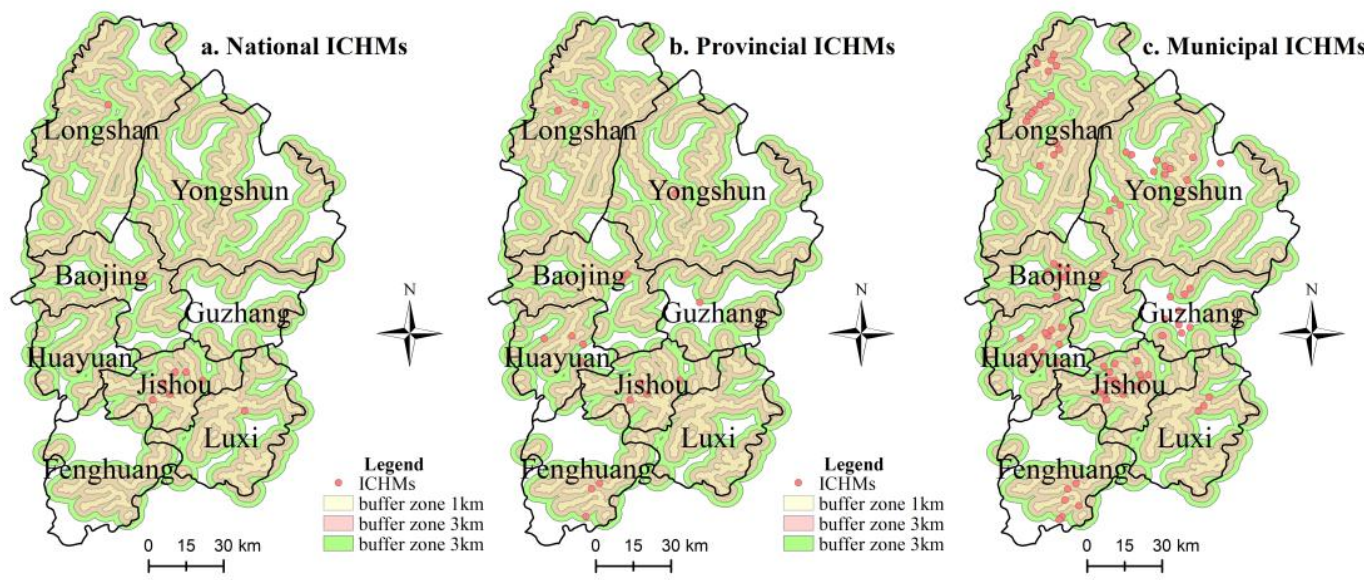

Fig. 5. The ICHMs and river multistage buffer zones in Xiangxi

Table 3. The ICHMs statistics of multilevel buffer zones in Xiangxi

\begin{tabular}{ccccccc}
\hline \multirow{2}{*}{$\begin{array}{c}\text { Buffer } \\
\text { zones }\end{array}$} & \multicolumn{2}{c}{ National ICHMs } & \multicolumn{2}{c}{ Provincial ICHMs } & \multicolumn{2}{c}{ Municipal ICHMs } \\
\cline { 2 - 7 } $0-1 \mathrm{~km}$ & 3 & $33.33 \%$ & 7 & $38.89 \%$ & 30 & $35.71 \%$ \\
$1-3 \mathrm{~km}$ & 6 & $66.67 \%$ & 8 & $44.44 \%$ & 41 & $48.81 \%$ \\
$3-5 \mathrm{~km}$ & 0 & $0 \%$ & 3 & $16.67 \%$ & 13 & $15.48 \%$ \\
\hline
\end{tabular}

3.3.2 The influence of topography on the distribution of ICHMs

Xiangxi is a typical mountainous area with insufficient plain areas, and the ICHMs are mainly concentrated in hilly (200-500 m) and low mountain areas (500-1000 m). Combined with Table 4, the national ICHMs are mainly concentrated in hilly areas, with 7 items, accounting for $77.78 \%$ of the total. One item is located in the low mountainous area, accounting for $11.11 \%$ of the total. As for provincial ICHMs, 10 items are located in hilly areas and 7 items are located in low mountainous areas, accounting for $94.44 \%$ of the total. In addition, 41 projects of municipal ICHMs are located in hilly areas, and 36 projects are located in low mountainous areas, accounting for $91.67 \%$ of the total. As a whole, the distribution of ICHMs has the directivity of low altitude, and the numbers in different terrains varies significantly, which decrease with the increase of altitude.

Table 4. The ICHMs statistics of various terrains in Xiangxi

\begin{tabular}{ccccccc}
\hline \multirow{2}{*}{ Terrains } & \multicolumn{2}{c}{ National ICHMs } & \multicolumn{2}{c}{ Provincial ICHMs } & \multicolumn{2}{c}{ Municipal ICHMs } \\
\cline { 2 - 7 } & quantity & proportion & quantity & proportion & quantity & proportion \\
\hline Plain & 1 & $11.11 \%$ & 1 & $5.56 \%$ & 3 & $3.57 \%$ \\
Hill & 7 & $77.78 \%$ & 10 & $55.56 \%$ & 41 & $48.81 \%$ \\
Low mountain & 1 & $11.11 \%$ & 7 & $38.88 \%$ & 36 & $42.86 \%$ \\
Middle mountain & 0 & $0 \%$ & 0 & $0 \%$ & 4 & $4.76 \%$ \\
\hline
\end{tabular}

Note: $0-200 \mathrm{~m}$ is plain, $200-500 \mathrm{~m}$ is hill, $500-1000 \mathrm{~m}$ is low mountain and $1000-3500 \mathrm{~m}$ is 
middle mountain.

\subsubsection{The influence of transportation on the distribution of ICHMs}

Transportation, which promotes population agglomeration and has an important impact on human production and life, is an important factor affecting the distribution of ICHMs. According to Fig. 6, the distribution of ICHMs in Xiangxi has traffic directivity. National ICHMs are distributed at the intersection of or along the main highways (Fig. 6a). The distribution of most provincial ICHMs is consistent with the national ICHMs, that is, at the intersection of or along the main highways, while a small amount is distributed along the railways (Fig. 6b). Municipal ICHMs are also distributed along traffic lines (highways, ordinary roads or railways), and they are particularly dense at the intersections of traffic lines (Fig. 6c). On the whole, highways have the greatest impact on ICHMs distribution, followed by railways and ordinary roads.
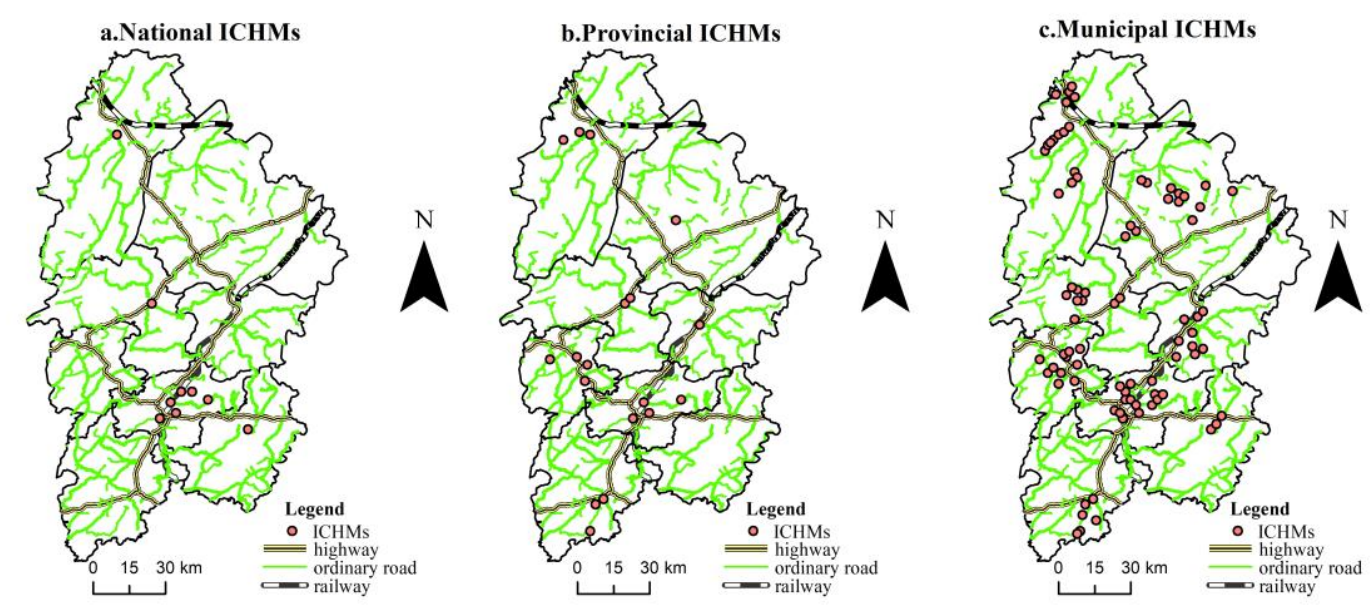

Fig. 6. The overlay map of traffic lines and ICHMs in Xiangxi

\subsubsection{The influence of economy on the distribution of ICHMs}

According to Fig. 7 (the abscissa is the quantity of ICHMs, and the ordinate is GDP), the economic development level of Xiangxi is closely related to the spatial distribution of ICHMs. Jishou, Yongshun and Longshan, in the first quadrant, belong to "double high" areas; that is, the number of ICHMs and GDP, which are positively correlated, are greater than the regional average level. Luxi, Baojing and Guzhang, with backward economies and fewer ICHMs in the third quadrant, are "double low" areas; that is, the number of ICHMs and GDP are both lower than the regional average level. Fenghuang in the second quadrant and Huayuan in the fourth quadrant are the type of one high while another low; in other words, the economic development level of the two regions is negatively correlated with the number of ICHMs. In general, the empirical tests of the most administrative units (75\%) show that the more developed the economy, the more ICHM projects, and vice versa. Therefore, the economy has a significant positive impact on ICHM programs. 


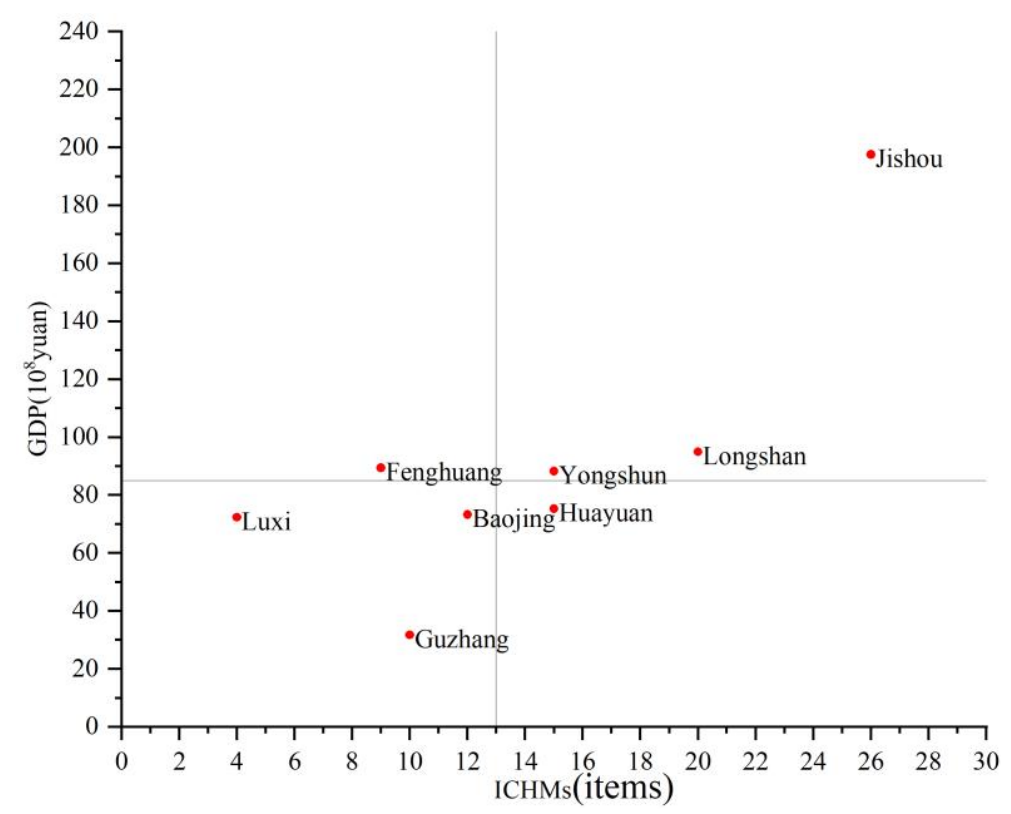

Fig. 7. The matrix analysis of GDP and ICHMs in Xiangxi

Note: the number of ICHMs is the sum of national, provincial and municipal ICHMs

\section{Discussion and conclusions}

\subsection{Discussion}

\subsubsection{Comparison with previous studies}

In this study, we aimed to analyze the spatial distribution characteristics of ICHMs and explore the main factors affecting their distribution in Xiangxi, Central China. Xiangxi was chosen as an empirical case, as it is an ethnic minority area, a mountainous area and a national cultural protection experimental area; moreover, it contains rich ICH resources. Methods such as the nearest neighbor index, kernel density estimation, and buffer analysis were used to ensure that the aforementioned problems were comprehensive and deeply explored.

The structure of ICHMs reflects that there is a lack of high-level ICHMs and quyi in Xiangxi. The quantities of national, provincial and municipal ICHMs in Xiangxi were 9, 18 and 84, respectively, implying that the higher the level, the lower the quantity, and vice versa. The results of this research are consistent with those of Bsa, $\mathrm{Mk}$ and $\mathrm{Cn}$ [25]. At the same time, quyi is the type of the least number, with 14 items in total, accounting for less than $13 \%$ of the total. The reason is that the expressive, demonstrative, experiential, and participation of quyi are not as good as other types of ICHM; as a result, its inheritance and protection are more difficult.

The results signify that the distribution of ICHMs has strong spatial heterogeneity, which is consistent with the results of Yao et al. [10] and Marzeion and Levermann [26]. In the research of Yao et al. (2021) [10], 81.9\% of Christian cultural heritage is 
concentrated in Europe, while investigators [26] have confirmed that world cultural heritage sites are concentrated near the coasts. In this study, most ICHMs are concentrated distributions, and a few are scattered distributions or random distributions. Meanwhile, the national ICHMs, provincial ICHMs and municipal ICHMs are mainly concentrated in the south, west, and central and northern regions, respectively. In addition, there are spatial differences in the density of various ICHMs. For example, the density of traditional music is characterized by three subhigh-density areas surrounding one high-density area.

In terms of the main influencing factors, topography, rivers, transportation and GDP had the most profound impact on the distribution of ICHMs; this outcome was consistent with the results of previous studies [1, 10,26,27]. In the research of Cho and Sung [1] and Marzeion and Levermann [26], ICHs are mainly concentrated in areas with flat terrain and sufficient water sources, such as coastal areas or plains. The results of Yao et al. [10] showcase that there is a certain correlation between economic development and the distribution of ICH. They have argued that the protection of ICH certainly requires financial support, and the more developed the economy, the more sufficient the financial support. In addition, it is well known that economic development is closely related to traffic accessibility, and they have a significant positive correlation. Therefore, traffic accessibility is also an important factor affecting the distribution of ICH [27].

In general, the results of this study are consistent with the findings reported by previous research, and they are also scientific and reasonable.

\subsubsection{Theoretical contributions and practical implications}

The results of this study provide a reference for future theoretical research on $\mathrm{ICH}$. Geographic methods, such as spatial analysis and spatial mapping, can realize the visual expression of spatial information and have been widely used in many fields [28-31]. At present, the application of geographical methods in the research of ICHs is still lacking. To this end, geographic methods were used to explore the spatial distribution characteristics and influencing factors of ICHMs in this study. The research belongs to the cross research category of geography and art, and the approaches used in this study can provide an effective tool for related research.

This study provides references for the management and protection of $\mathrm{ICH}$. On the one hand, the results showcase the sparsity of quyi, so it is of great importance for its rational protection and effective utilization. On the other hand, economic development contributes to the protection of ICH. At the same time, ICH is also an important tourism resource. The development of ICH tourism is an important direction that can not only realize the sustainable development of the economy but also promote the protection of 
ICH.

\subsubsection{Future research}

Xiangxi is a minority area, and the ethnic composition of each research unit varies little (the proportion of ethnic minorities for all units is greater than $85 \%$ ), so this study does not consider the impact of ethnic composition on the distribution of ICHM. Nevertheless, there are 56 ethnic groups in China, and the ethnic composition varies greatly in different regions. Therefore, the applicability of the results of this study in nonminority areas remains to be verified. Therefore, the comparison between ethnic minority areas and nonethnic minority areas to explore the impact of ethnic composition on the distribution of ICH is an important direction of in-depth research in the future.

\subsection{Conclusions}

The main conclusions of this study are as follows: 1) traditional music, traditional dance and traditional drama are abundant, while quyi is relatively lacking in Xiangxi. The numbers of traditional music, traditional dance, traditional drama and quyi are 33, 34,30 and 14, respectively, and the number of quyi is less than half that of other categories. 2) The distribution of ICHMs in Xiangxi has spatial heterogeneity, and the main trend is agglomeration. The national ICHMs, provincial ICHMs and municipal ICHMs are mainly concentrated in the south, the west, and the central and northern regions, respectively. The high-density area of traditional music is located in the south, of traditional drama in the west, and of quyi in the north. 3) The distribution of ICHMs is affected by four factors: namely rivers, topography, GDP and transportation. The 0 $3 \mathrm{~km}$ buffer zone of the rivers, with $100 \%$ of national ICHMs, $83.33 \%$ of provincial ICHMs and $84.52 \%$ of municipal ICHMs, is the centralized distribution area of ICHMs. $100 \%$ of national ICHMs, $94.44 \%$ of provincial ICHMs and $91.67 \%$ of municipal ICHMs are distributed in hilly (200-500 m) and low mountain areas (500-1000 m). Highways have the greatest impact on ICHM distribution, followed by railways and ordinary roads. The economy has a significant positive impact on ICHM programs; the more developed the economy, the more ICHM projects.

Author Contributions: conceptualization, X-Y. Z, H. X, and R.L.; data collection: XY. Z; formal analysis, X-Y. Z, H. X, and R.L.; funding acquisition, X-Y. Z.; investigation, X-Y.Z.; methodology, X-Y. Z, H. X, and R.L.; original draft, X-Y. Z, and H.X.; review and editing, X-Y. Z, H. X, and R.L.

Funding: This work was supported by the educational science planning project of 
Hunan Province (Grant XJK21CTW001) and the research project on educational reform of degree and graduate education of Jishou University.

Acknowledgments: We would like to thank employees who work in government departments in Xiangxi for their valuable suggestions and datasets. We also acknowledge the financial support from the research group.

Conflict of interest: The authors of this article declare no potential conflicts of interest. Availability of data and materials: The datasets used and/or analyzed during the current study are available from the corresponding author on reasonable request.

\section{References}

[1] Cho, s. Sung: A Study on the Characteristics of Cultural Spaces associated with Intangible Cultural Heritage, The Study of Practice Folkloristics, 30 (2017) 155186.

[2] L. Federico: Intangible Cultural Heritage: The Living Culture of Peoples, Eur. J. Int. Law, (2011) 101-120.

[3] Beardslee, Thomas: Whom does heritage empower, and whom does it silence? Intangible cultural heritage at the Jemaa el Fnaa, Marrakech, International Journal of Heritage Studies, (2015) 1-13.

[4] Craith, Mairead, Nic: UNESCO, Cultural Heritage, and Outstanding Universal Value: Value-Based Analyses of the World Heritage and Intangible Cultural Heritage Conventions, Amer. Anthrop., 3 (2013) 528-530.

[5] C. Sun, H. Chen, R. Liao: Research on Incentive Mechanism and Strategy Choice for Passing on Intangible Cultural Heritage from Masters to Apprentices, Sustainability, 13 (2021) 5245.

[6] Tan, Siow-Kian, Siow-Hooi, Kok, Yon-Sin, Choon, Shay-Wei: Sense of place and sustainability of intangible cultural heritage - The case of George Town and Melaka, Tourism Management, 67 (2018) 376-387.

[7] L. Zhuang: Indigenising Intangible Cultural Heritage: Comparison and Interpretation of the Concept of ICH in China, International Journal of Intangible Heritage, 10 (2015) 125-134.

[8] M. 0tt, F.M. Dagnino, F. Pozzi: Intangible Cultural Heritage: Towards collaborative planning of educational interventions, Comput. Human Behav., 51 (2015) 1314-1319.

[9] X. Su, X. Li, Y. Wu, L. Yao: How Is Intangible Cultural Heritage Valued in the Eyes of Inheritors? Scale Development and Validation, Journal of Hospitality \& Tourism Research, 44 (2020) 91469.

[10] Y. Yao, X. Wang, L. Lu, C. Liu, Q. Wu, H. Ren, S. Yang, R. Sun, L. Luo, K. Wu: Proportionated Distributions in Spatiotemporal Structure of the World Cultural Heritage Sites: Analysis and Countermeasures, Sustainability, 13 (2021) 2148.

[11] X. Liu, M. Li: Safeguarding intangible cultural heritage to promote mental healthcare in China: Challenges to maintaining the sustainability of safeguarding efforts, Int. J. Soc. Psychiatry, 66 (2020) 1-3.

[12] K. L. Chen, M. F. Ahmed, M. B. Mokhtar, K. L. Tan, M. Z. Idris, C. C. Yi: Understanding 
Intangible Culture Heritage Preservation via Analyzing Inhabitants' Garments of Early 19th Century in Weld Quay, Malaysia, Sustainability, 13 (2021) 5393.

[13] S. Tzima, G. Styliaras, A. Bassounas, M. Tzima: Harnessing the Potential of Storytelling and Mobile Technology in Intangible Cultural Heritage: A Case Study in Early Childhood Education in Sustainability, Sustainability, 12 (2020) 9416.

[14] A. Pommerening, J. Szmyt, G. Zhang: A new nearest-neighbour index for monitoring spatial size diversity: The hyperbolic tangent index, Ecol. Model., 435 (2020).

[15] M. Kück, E. M. Fr: Forecasting of customer demands for production planning by local k-nearest neighbor mode1s, Int. J. Product. Econ., 231 (2021).

[16] C. Minoiu, S.G. Reddy: Kernel density estimation on grouped data: the case of poverty assessment, The Journal of Economic Inequality, 6 (2014) 55-74.

[17] C. Minoiu, S. G. Reddy: Kernel Density Estimation Based on Grouped Data: The Case of Poverty Assessment, Social Science Electronic Publishing, 12 (2014) 163-189. [18] Z. M. Zhanga, X. Y. Wanga, Y. Zhanga, Z. Nana, B. G. Shenb: The Over Polluted Water Quality Assessment of Weihe River Based on Kernel Density Estimation, Procedia Environmental Sciences, 13 (2012) 1271-1282.

[19] J. Jin, L. Geert: Identifying Outliers in Response Quality Assessment by Using Multivariate Control Charts Based on Kernel Density Estimation, Journal of Official Statistics, 37 (2021) 77-84.

[20] Y. Zheng, X. Zhao, L. Yao: Mixture Kernel Density Estimation and Remedied Correlation Matrix on the EEG-Based Copula Model for the Assessment of Visual Discomfort, Cognit. Comput., 12 (2020) 1130-1143.

[21] S. Panda, P. K. Nanda: Kernel density estimation and correntropy based background modeling and camera model parameter estimation for underwater video object detection, Soft Computing, 25 (2021) 10477-10496.

[22] A. Aboubacar, M.E. Machkouri: Recursive kernel density estimation for time series, ITIT, 99 (2020) 1-15.

[23] K. Saito, M. Yano, H. Hino, T. Shoji, A. Asahara, H. Morita, C. Mitsumata, J. Kohlbrecher, K. Ono: Accelerating small-angle scattering experiments on anisotropic samples using kernel density estimation, Sci. Rep., 9 (2019) 112-128.

[24] M. Deng, X. Yang, Y. Shi, J. Gong, Y. Liu, H. Liu: A density-based approach for detecting network-constrained clusters in spatial point events, International Journal of Geographical Information Science, 33 (2019) 466-488.

[25] B. Bsa, B. Mk, A. Cn: Heritage Impact Assessment, beyond an Assessment Tool: A comparative analysis of urban development impact on visual integrity in four UNESCO World Heritage Properties - ScienceDirect, Journal of Cultural Heritage, 8 (2020) 3818

[26] B. Marzeion, A. Levermann: Loss of cultural world heritage and currently inhabited places to sea-level rise, ENVIRON RES LETT, 9 (2014) 2033-2053.

[27] J. J. Wang: Flood risk maps to cultural heritage: Measures and process, Journal of Cultural Heritage, 16 (2015) 210-220.

[28] S. Ali, H. Ali, M. Pakdel, S. G. Askari, A. A. Mohammadi, S. Rezania: Spatial analysis and probabilistic risk assessment of exposure to fluoride in drinking water using GIS and Monte Carlo simulation, Environmental Science and Pollution Research, 
7 (2021) $1-10$.

[29] H. Wang, Y. Jiang, B. Li, Y. Cui, R. Li: Single-Cell Spatial Analysis of Tumor and Immune Microenvironment on Whole-Slide Image Reveals Hepatocellular Carcinoma Subtypes, Cancers (Base1), 12 (2020) 3562.

[30] U. Akram, N. H. Qu Tt Ineh, U. Wennergren, K. Tonderski, G. S. Metson: Author Correction: Enhancing nutrient recycling from excreta to meet crop nutrient needs in Sweden - a spatial analysis, Sci. Rep., 10 (2020) 361.

[31] K. Berzins, S. J. Fraser-Miller, K. C. Gordon: A New Frontier for Nondestructive Spatial Analysis of Pharmaceutical Solid Dosage Forms: Spatially Offset Low-Frequency Raman Spectroscopy, AnaCh, 93 (2021) 3698-3705. 